\title{
Aesthetic Curve Design with Linear Gradients of Logarithmic Curvature/Torsion Graphs
}

\author{
R. U. Gobithaasan ${ }^{1}$, Kenjiro T. Miura ${ }^{2}$, L. P. Yee ${ }^{1} \&$ A. F. Wahab ${ }^{1}$ \\ ${ }^{1}$ School of Informatics \& Applied Mathematics, University Malaysia Terengganu, Kuala Terengganu, Malaysia \\ ${ }^{2}$ Graduate School of Science and Technology, Shizuoka University, Shizuoka, Japan \\ Correspondence: R. U. Gobithaasan, School of Informatics \& Applied Mathematics, University Malaysia \\ Terengganu, 21030 Kuala Terengganu, Malaysia. Tel: 60-9-668-3534. E-mail: gr@umt.edu.my
}

Received: February 27, $2014 \quad$ Accepted: March 25, $2014 \quad$ Online Published: April 8, 2014

doi:10.5539/mas.v8n3p24

URL: http://dx.doi.org/10.5539/mas.v8n3p24

\begin{abstract}
The quality of a curve for industrial design and computer graphics can be interrogated using Logarithmic Curvature Graph (LCG) and Logarithmic Torsion Graph (LTG). A curve is said to be aesthetic if it depicts linear LCG and LTG function. The Log-aesthetic curve (LAC) was developed bearing this notion and it was later extended to a Generalized Log-aesthetic curve (GLAC) using the $\kappa$-shift and $\rho$-shift approach. This paper reformulates GLAC by representing the Logarithmic Curvature and Torsion graph's gradient function as a nonlinear ordinary differential equation (ODE) with boundary conditions. The outputs of solving the ODEs result in a well defined Cesaro equation in the form of curvature function that is able to produce both planar as well as spatial curves with promising entities for industrial product design, computer graphics and more.
\end{abstract}

Keywords: visually pleasing curves, fair curves, aesthetic curves, Computer Aided Design

\section{Introduction}

\subsection{The Formulation of Logarithmic Curvature Graph That Leads to Log-Aesthetic Curves}

Designing visually pleasing industrial products is crucial since this feature dicates the success of a product (Pugh, 1991). Harada et al. (1999) proposed a novel method to investigate curves used in automobile design called the Logarithmic Distribution Diagram of Curvature (LDDC). They proposed aesthetic curves as curves with a constant LDDC gradient where the gradient is denoted as $\alpha$. In 2003, Kanaya et al. simplified the formulation of LDDC to a simpler form, hence denoting it as Logarithmic Curvature Graph (LCG). Recently, Gobithaasan and Miura (2014) further proposed on using LCG as a shape interrogation tool for arbitrary curves.

In 2005, Miura derived the fundamentals used to design aesthetic curves and consequently developed a general formula of aesthetic curves called Log-Aesthetic Curves (LAC). Yoshida and Saito (2006) further proposed a method to draw the Log-Aesthetic Curve segment interactively by using two endpoints and their respective tangent vectors, known as the $\mathrm{G}^{1}$ data. In 2009, Levien and Sequin stated that the Log Aesthetic curve is the most promising curve for aesthetic design. In 2012, Yoshida and Saito further derived a method to render the drawable boundary for Log-Aesthetic Curve segments to indicate whether a segment can be drawn from the given $\mathrm{G}^{1}$ data or otherwise.

\subsection{Generalized Log-Aesthetic Curve}

In 2011, Gobithaasan and Miura proposed a planar curve called Generalized Log Aesthetic Curve (GLAC) by extending the formulation of Generalized Cornu Spiral (GCS) (Ali et al., 1999) in a similar manner to Log-Aesthetic Curve. The GLAC comprises of high quality planar spirals such as Generalized Cornu Spiral, Log-Aesthetic Curve, clothoid, Nielsen's spiral, logarithmic spiral, circle involute and etc. It has an additional parameter (denoted as $v$ ) to determine its shape. Compared to the Generalized Cornu Spiral, the Generalized Log Aesthetic Curve segment can be used to satisfy an extra constraint during the design process. To date, it has been further extended to the 3D GLAC called Generalized Log Aesthetic Space Curve (GLASC) (Gobithaasan et al., 2012). 


\subsection{Recent Advancement of Log Aesthetic Curve}

The Log Aesthetic Curve has attracted numerous researchers around the world to investigate its practical applications. Readers are referred to a comprehensive review of these curves by Miura and Gobithaasan (2014) and references therein. In 2013, an article published in Computer Aided Design and Application journal entitled "Designing $G^{2}$ Log-Aesthetic Spline" was selected as the best overall paper during the $10^{\text {th }}$ International Conference of Computer Aided Design \& Application held in Bergamo, Italy. This paper highlights the readiness of Log Aesthetic Curve for practical Computer Aided Design application pertaining to designing $\mathrm{G}^{2}$ continuous LAC in automobile design (Miura et al., 2013).

\subsection{Research Highlight}

In order to indicate its robustness in Computer Aided Design applications, Gobithaasan et al. (2013) showed that the Generalized Log Aesthetic Curve has wider drawable region compared to Log Aesthetic Curve. However, the

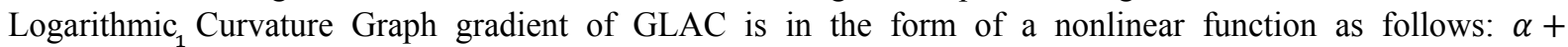
$v(\Lambda \alpha s+1)^{\frac{1}{\alpha}}(1+\alpha)$ whereas LAC has LCG gradient as $\alpha$. The LCG gradient of GLAC may consist of two segments with opposite sign of LCG gradient, known as the compound rhythm LAC. The highlight of this paper is that we re-formulate the Generalized Log Aesthetic Curve. The final output indicates that the curvature is in a simpler form as compared to the original Generalized Log Aesthetic Curve and the new 2D and 3D GLACs preserve the linearity of Logarithmic Curvature Graph.

\section{Logarithmic Curvature and Torsion Graph's Gradient}

Gobithaasan et al. (2009) proposed the formula of the Logarithmic Curvature Graph's gradient as follows:

$$
\lambda_{\kappa}(s)=1-\frac{\rho(s) \rho^{\prime \prime}(s)}{\rho^{\prime}(s)^{2}}
$$

From Equation (1), we know that when the first derivation of the curvature radius equals to zero, hence Equation (1) cannot be determined. For the circle and straight line, only the first derivation of curvature radius equals to zero. A straight line has no radius of curvature hence it does not have both LCGand corresponding gradient. In the case of a circle, the radius of curvature is a constant value and arc length is $2 \pi A$, where $\mathrm{A}$ is the radius of a circle. Hence, the LCG gradient should be

$$
\lambda_{\kappa}(t)=\frac{\frac{d \log \left[\rho(t) \frac{\mathrm{ds}}{\mathrm{d} \rho}\right]}{d t}}{\frac{d \log [(t)]}{d t}}=\frac{d \log [A(2 \pi)]}{d \log [\mathrm{A}]}=0
$$

Similarly, the Logarithmic Torsion Graph's gradient proposed by Gobithaasan et al. (2012) is

$$
\lambda_{\tau}(s)=1-\frac{\mu(s) \mu \prime \prime(s)}{\mu \prime(s)^{2}}
$$

Equation (3) is not available for helix due to its constant torsion profile, so the LTG gradient for helix, $\lambda_{\tau}=0$.

\subsection{Curvature Profile from Linear Logarithmic Curvature Graph's Gradient}

Equation (1) can be modified with regards to the curvature function, $\kappa(s)$ as

$$
\lambda_{\kappa}=\frac{\kappa^{\prime \prime}(\mathrm{s}) \kappa(\mathrm{s})}{\kappa^{\prime}(\mathrm{s})^{2}}-1
$$

Therefore, a linear LCG gradient setting is

$$
\frac{\kappa^{\prime \prime}(\mathrm{s}) \kappa(\mathrm{s})}{\kappa^{\prime}(\mathrm{s})^{2}}-1=\gamma s+\alpha
$$

where $\gamma$ represents the gradient in LCG and $\alpha$ is a constant value. Solving the second order nonlinear differential Equation (5) results in

$$
\kappa(s)=\left\{\begin{array}{cc}
\mathrm{c}_{2} e^{\mathrm{c}_{1} s} & \text { if } \alpha=\gamma=0 \\
\mathrm{c}_{2}\left(\mathrm{c}_{1}+s \alpha\right)^{-1 / \alpha} & \text { if } \gamma=0 \\
2 \operatorname{ArcTan}\left[\frac{\alpha+s \gamma}{\sqrt{-\alpha^{2}+2 c_{1} \gamma}}\right] & \\
-\frac{\sqrt{-\alpha^{2}+2 c_{1} \gamma}}{c_{2} e} & \text { otherwise }
\end{array}\right.
$$

where $c_{1}$ and $c_{2}$ are constants solved from differential equation. Equation (5) is rearranged to become

$$
\kappa^{\prime \prime}(\mathrm{s})=\frac{(1+\alpha+s \gamma) \kappa^{\prime}(\mathrm{s})^{2}}{\kappa(\mathrm{s})}
$$


where $0 \leq s \leq S, S$ is the total arc length. Let

$$
f\left(s, \kappa, \kappa^{\prime}\right)=\frac{(1+\alpha+s \gamma) \kappa^{\prime}(\mathrm{s})^{2}}{\kappa(\mathrm{s})}
$$

for $0 \leq s \leq S$, with $\kappa(0)=\kappa_{a}$ and $\kappa(\mathrm{S})=\kappa_{b} . f\left(s, \kappa, \kappa^{\prime}\right)$ is continuous on the set $D=\left\{\left(s, \kappa, \kappa^{\prime}\right) \mid\right.$ for $0 \leq s \leq S$, with $-\infty<\kappa<\infty$ and $\left.-\infty<\kappa^{\prime}<\infty\right\}$. The partial derivatives are $f_{\kappa}\left(s, \kappa, \kappa^{\prime}\right)=-\frac{(1+\alpha+s \gamma) \kappa^{\prime}(s)^{2}}{\kappa(s)^{2}}$ and $f_{\kappa^{\prime}}\left(s, \kappa, \kappa^{\prime}\right)=\frac{2(1+\alpha+s \gamma) \kappa^{\prime}(\mathrm{s})}{\kappa(\mathrm{s})}$, where both are continuous on D. According to Keller (1968), the first condition for an unique solution to exist is, a constant M must exist so that $\left|f_{\kappa^{\prime}}\left(s, \kappa, \kappa^{\prime}\right)\right|=\left|\frac{2(1+\alpha+S \gamma) \kappa^{\prime}(s)}{\kappa(s)}\right| \leq \mathrm{M}$. The second condition which must be satisfied is $f_{\kappa}\left(s, \kappa, \kappa^{\prime}\right)=-\frac{\left(1+\alpha+s \gamma \kappa^{\prime}(\mathrm{s})^{2}\right.}{\kappa(s)^{2}}>0$ for all $\left(s, \kappa, \kappa^{\prime}\right) \in D$. Hence, these conditions can be used as a guide to ensure that Equation (7) has an unique solution based on the selections of $\{\alpha, \gamma\}$. For example, when $\alpha=-1$, the curve becomes clothoid in which a solution always exists when $\gamma<0$. In general, the inequality $\gamma<(-1-\alpha) / s$ must hold in order to ensure an unique solution always exist. Similar analysis can be carried out for torsion function to cofirm the existence of a solution.

In order to control the curvature's end point, Equation (6) is modified as

$$
\kappa(s)=\left\{\begin{array}{cc}
\left(\frac{\kappa_{1}}{c_{1}}\right)^{s / s} c_{1} & \text { if } \alpha=\gamma=0 \\
\kappa_{1}\left(s \alpha+c_{1}\right)^{-1 / \alpha}\left(S \alpha+c_{1}\right)^{\frac{1}{\alpha}} & \text { if } \gamma=0 \\
-\frac{2}{\sqrt{-\alpha^{2}+2 \gamma c_{1}}}\left(\operatorname{ArcTan}\left[\frac{\alpha+s \gamma}{\sqrt{-\alpha^{2}+2 \gamma c_{1}}}\right]-\operatorname{ArcTan}\left[\frac{\alpha+S \gamma}{\sqrt{-\alpha^{2}+2 \gamma c_{1}}}\right]\right)_{\kappa_{1}} & \text { otherwise }
\end{array}\right.
$$

where $\kappa_{1}=\kappa(S)$. To ensure that Equation (9) is always monotonic, there are constraints that need to be satisfied. These include, when $\gamma=0, \kappa_{1} \neq 0$ and has lower bound where $s>-\frac{c_{1}}{\alpha}$. In the case of $\gamma \neq 0$ and $\alpha \neq 0$, $2 \mathrm{c}_{1} \gamma>\alpha^{2}$ and $\kappa_{1} \neq 0$.

\subsection{Torsion Profile from Linear Logarithmic Torsion Graph's Gradient}

First, change Equation (5) with regards to torsion profile, $\tau(s)$ as

$$
\lambda_{\tau}=\frac{\tau^{\prime \prime}(\mathrm{s}) \tau(\mathrm{s})}{\tau^{\prime}(\mathrm{s})^{2}}-1
$$

Then, to obtain the torsion profile from linear LTG gradient, set

$$
\frac{\tau^{\prime \prime}(s) \tau(s)}{\tau^{\prime}(s)^{2}}-1=\psi s+\beta
$$

where $\psi$ represents the gradient of LTG and $\beta$ is just an arbitrary constant. Solving the second order nonlinear differential Equation (11) results in

$$
\tau=\left\{\begin{array}{cc}
\mathrm{d}_{2} e^{\mathrm{d}_{1} s} & \text { if } \beta=\psi=0 \\
\mathrm{~d}_{2}\left(\mathrm{~d}_{1}+s \beta\right)^{-1 / \beta} & \text { if } \psi=0 \\
2 \operatorname{ArcTan}\left[\frac{\beta+s \psi}{\sqrt{-\beta^{2}+2 \mathrm{~d}_{1} \psi}}\right] & \\
-\frac{\sqrt{-\beta^{2}+2 \mathrm{~d}_{1} \psi}}{\mathrm{d}_{2} e} & \text { otherwise }
\end{array}\right.
$$

where $d_{1}$ and $d_{2}$ are constants solved from the differential equation. Similar to the formulation of curvature profile, Equation (12) too has an unique solution. Equation (12) is modified to enable control of the torsion at end point

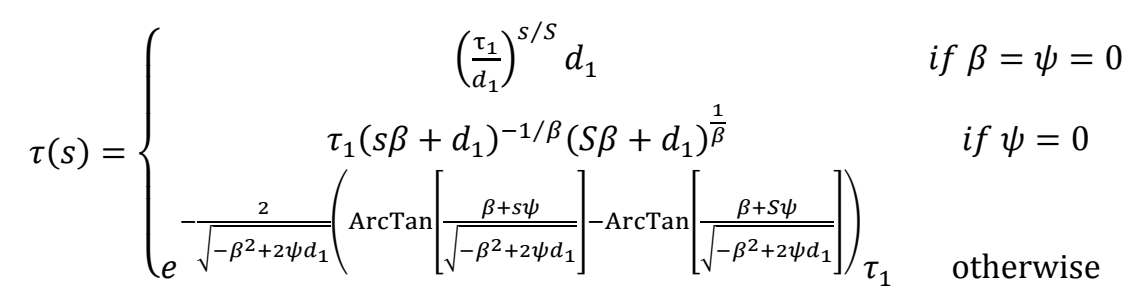

where $\tau_{1}=\tau(S)$. To ensure that Equation (13) is always monotonic, some constraints need to be followed. These conditions are, when $\psi=0, \tau_{1} \neq 0$ and has lower bound where $s>-\frac{d_{1}}{\beta}$. When $\psi \neq 0$ and $\beta \neq 0$, 
$2 \mathrm{~d}_{1} \psi>\beta^{2}$ and $\tau_{1} \neq 0$.

\section{The Construction of 2D and 3D Generalized Log Aesthetic Curve}

A planar curve is obtained when the curvature profile is solved using the Frenet-Serret equation without the torsion profile (Gray, 1994). For example, when $\kappa_{1}=1, \alpha=1, \gamma=1, c_{1}=1$ with various $S=\{1,2,3,4,5\}$. These are monotonic decreasing curvature profiles as shown in Figure 1 and thecurves are rendered in Figure 2. On the other hand, when values are changed i.e. $\gamma=-1$ and $c_{1}=-1$, these curvature profiles will increase monotonically and $\kappa_{1}=3$ as shown in Figure 3 and 4. Figure 5 shows the curvature profile with different end of curvature value with the same arc length, $2 \pi$ and corresponding curves are shown in Figure 6.

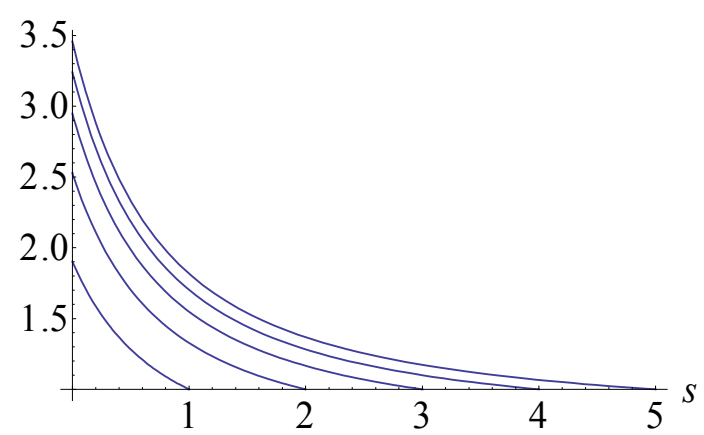

Figure 1. Monotonically decreasing curvature profile with arc length $s=\{1,2,3,4,5\}$

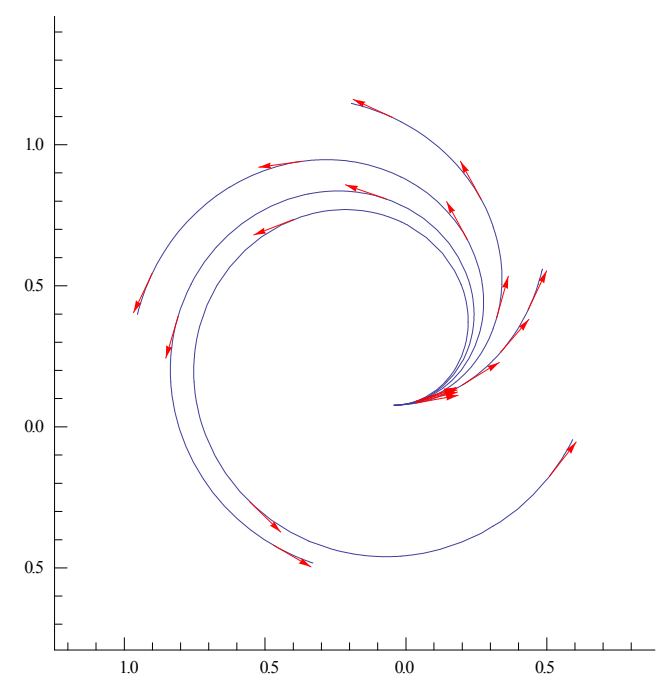

Figure 2. Five examples of planar curves with similar end curvatures and arc length $s=\{1,2,3,4,5\}$

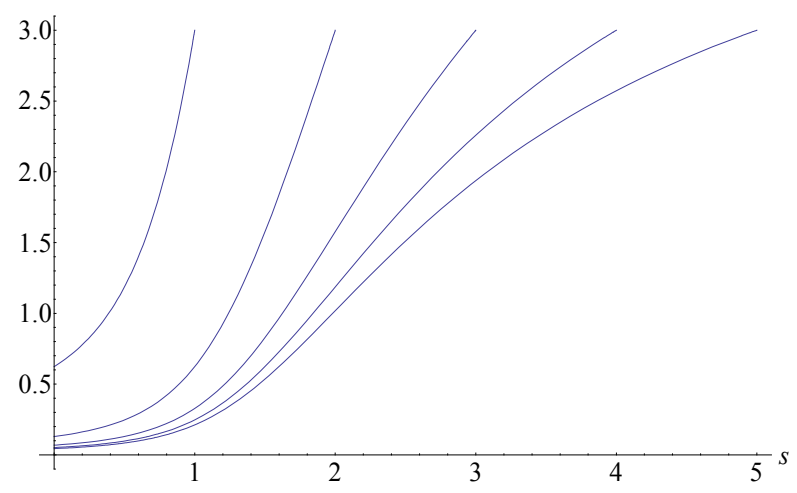

Figure 3. Monotonically increasing curvature profile with arc length $s=\{1,2,3,4,5\}$ 


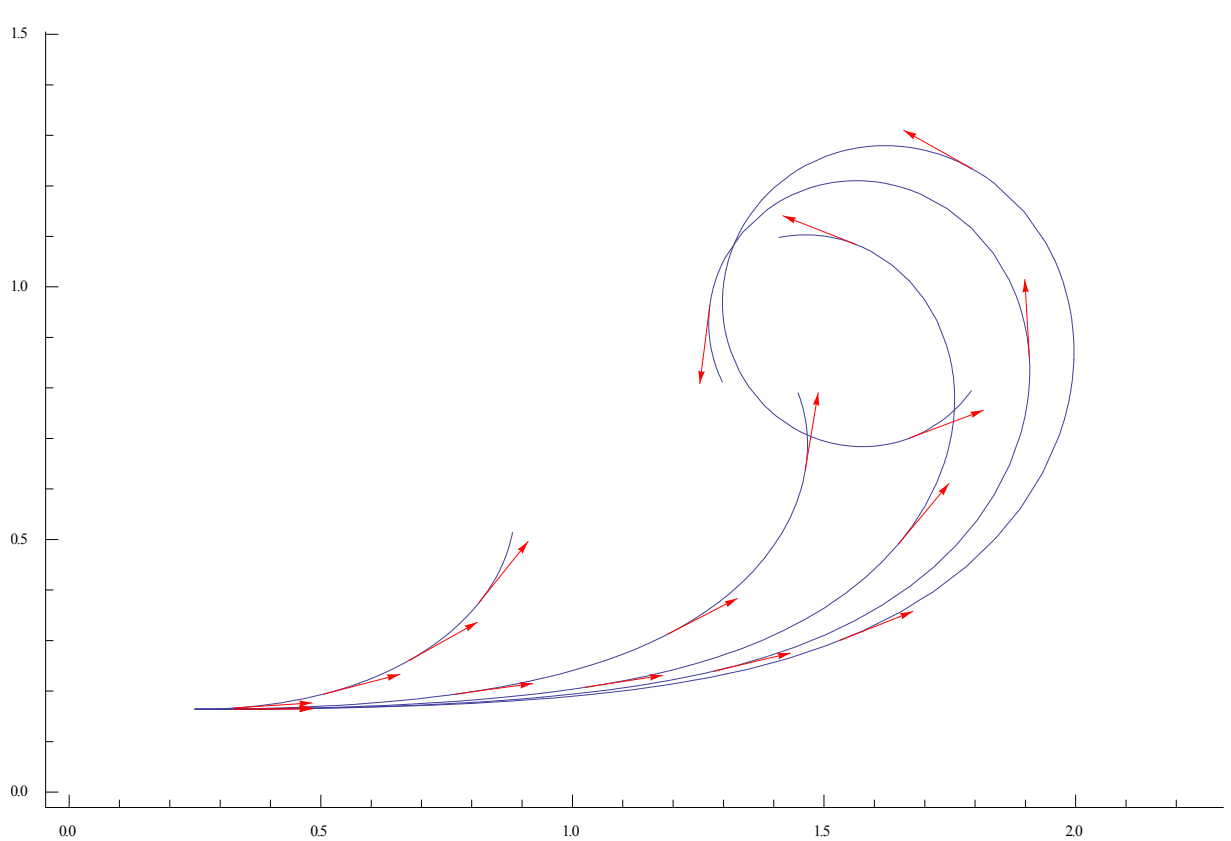

Figure 4. Examples of planar curves with similar curvature at end point with arc length $s=\{1,2,3,4,5\}$

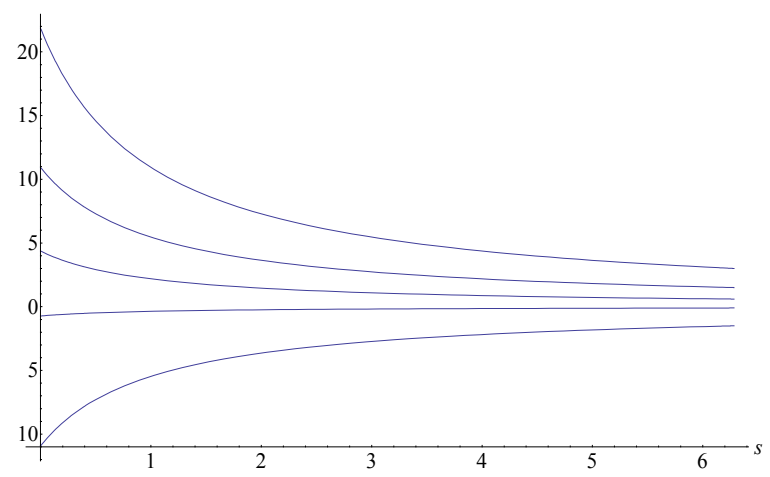

Figure 5. Curvature profile with different end curvatures

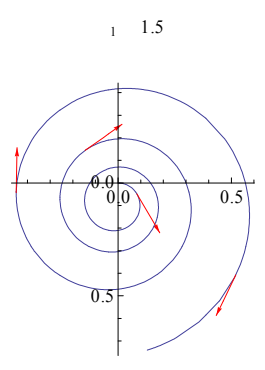

(a)

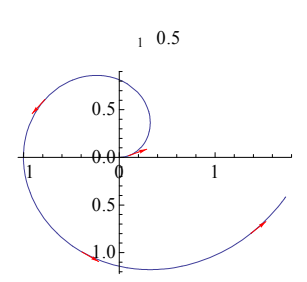

(b)

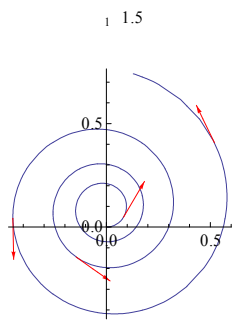

(c)

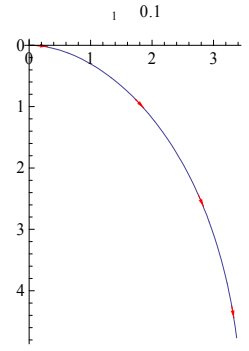

(d)

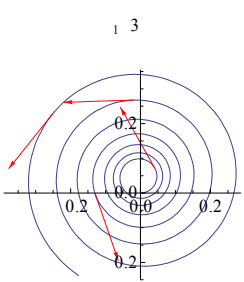

(e)

Figure 6. Examples of planar curve with various end curvatures but same arc length

The formulation of the torsion profile is done in the same way as the formulation of curvature profile. Therefore in Figure 7, the pattern of curvature profile of Figure 4 is applied into the torsion profile. Figure 8 depicts the pattern of Figure 6's curvature profile when applied into the torsion profile. 


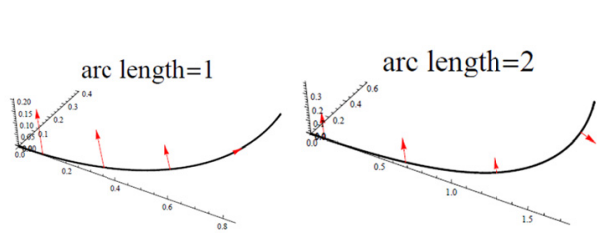

(a)

(b)

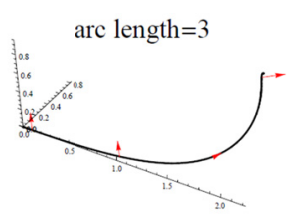

(c)

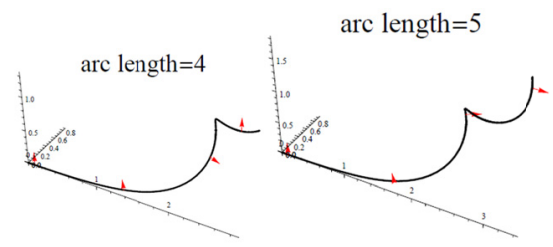

(d)

(e)

Figure 7. Planar curves with similar curvatures and torsions at end point with arc length $s=\{1,2,3,4,5\}$

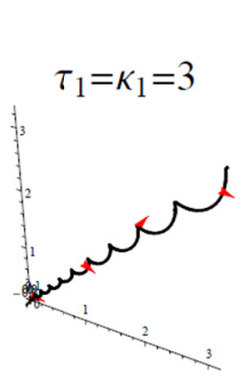

(a)

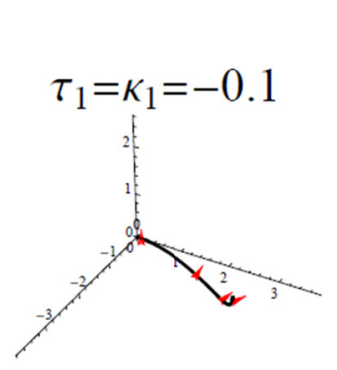

(b)

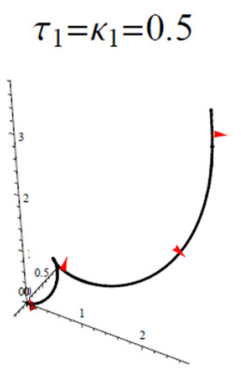

(c)

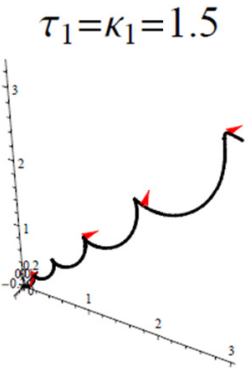

(d)

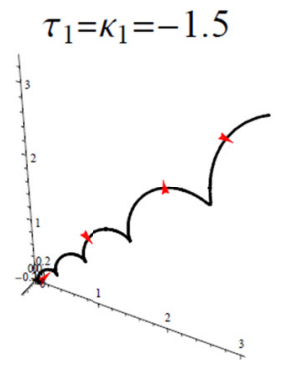

(e)

Figure 8. Space curves with five types of similar curvatures and torsions at end point with the same arc length

\section{A Note of Revised Generalized Log Aesthetic Curve}

When $c_{1}=\kappa_{1}$ and $\gamma=\alpha=0, \kappa=c_{1}$ is obtained, which is the constant curvature profile for a circle. When a similar situation occurs to the torsion profile where $d_{1}=\tau_{1}$ and $\psi=\beta=0$, a helix is obtained. Additionally when $\kappa_{1}=0$ and $\tau_{1}=0$ then $\kappa=\tau=0$, hence a straight line is obtained.

The Log Aesthetic Curye has a constant value of Logarithmic Curvature Graph's gradient, hence when $\kappa=$ $\left(c_{1}+s \alpha\right)^{-1 / \alpha}\left(c_{1}+S \alpha\right)^{\bar{\alpha}} \kappa_{1}$, it becomes the curvature profile for LAC. The parameter $\alpha$ refers to the gradient in LCG as LAC. Due to this, $\tau=\left(\mathrm{d}_{1}+s \beta\right)^{-1 / \beta}\left(\mathrm{d}_{1}+S \beta\right)^{\bar{\beta}} \tau_{1}$ is also defined as the torsion profile for Log Aesthetic Space Curve since it has a linear profile of LTG with $\beta$ as gradient.

In Generalized Log Aesthetic Space Curve, there are some cases when theLCG and LTG gradients are in a linear form. The linear form of LCG and LTG gradient in GLASC are defined as

$$
\begin{aligned}
& \lambda_{\kappa}=1-s \Lambda+v \\
& \lambda_{\tau}=\zeta+\frac{1}{\omega}-s \Omega
\end{aligned}
$$

In conclusion, compared to the shape parameters derived, note that when $\gamma=-\Lambda$ and $\alpha=1+\nu$ the original Generalized Log Aesthetic Curve is derived, and when $\psi=-\Omega$ and $\beta=\zeta+\frac{1}{\omega}$ the original GLASC is also derived.

\section{Conclusion}

This paper reformulates Generalized Log Aesthetic Curve with linear Logarithmic Curvature and Torsion Graphs as shown in Equation (5) and (11). The curvature/torsion of revised GLACs is in a simpler form and the end curvatures/torsions can also be controlled to design $G^{2}$ continuous spline similar to the works of Ahmad et al. (2007) and Gobithaasan \& Ali (2004). Future works include the overall shape analysis and interactive control as conducted by Yoshida and Saito (2006) for practical Computer Aided Design applications.

\section{Acknowledgements}

The authors acknowledge University Malaysia Terengganu and Ministry of Education Malaysia (FRGS: 59265) for providing financial aid to carry out this research.

\section{References}

Ahmad, A., Gobithasan, R., \& Ali, J. Md. (2007). G2 transition curve using quartic Bezier curve, Computer Graphics, Imaging and Visualization: New Advances, CGIV 2007, 223-228. http://dx.doi.org/10.1109/CGIV.2007.44 
Ali, J. M., Tookey, R. M., Ball, J. V., \& Ball, A. A. (1999). The generalised Cornu spiral and its application to span generation. Journal of Computational and Applied Mathematics, 102, 37-47.

Farin, G. E. (1997). Curves and Surfaces for Computer Aided Geometric Design (5th ed.). USA: Academic Press.

Gobithasan, R., \& Ali, J. M. (2004). Towards G2 curve design with Timmer Parametric Cubic, Int. Conf. on Computer Graphics, Imaging and Visualization, CGIV 2004, 109-114. http://dx.doi.org/10.1109/CGIV.2004.1323969

Gobithaasan, R. U., Karpagavalli, R., \& Miura, K. T. (2013). Shape analysis of Generalized Log-Aesthetic curves, Int. Journal of Math. Analysis, 7(36), 1751-1759. http://dx.doi.org/10.1155/2013/732457

Gobithaasan, R. U., \& Miura, K. T. (2011). Aesthetic Spiral for Design. Sains Malaysiana, 40(11), 1301-1305.

Gobithaasan, R. U., \& Miura, K. T. (2014). Logarithmic Curvature Graph as a Shape Interrogation Tool. Applied Mathematical Sciences, 8(16), 755-765. http://dx.doi.org/10.12988/ams.2014.312709

Gobithaasan, R. U., Miura, K. T., \& Ali, J. M. (2009). The Elucidation of Planar Aesthetic Curves. 17th International Conference in Central Europe on Computer Graphics, Visualization and Computer Vision, WSCG'2009 - In Co-operation with EUROGRAPHICS, Full Papers Proceedings (pp. 183-188).

Gobithaasan, R. U., Yee, L. P., \& Miura, K. T. (2012). A Generalized Log Aesthetic Space Curve. ACM Proceedings of the 2012 Joint Int. Conf. on Human-Centered Computer Environments (pp. 145-149). http://dx.doi.org/10.1145/2160749.2160780

Gray, A. (1994). Modern Differential Geometry of Curves and Surfaces. Boca Raton, FL: CRC Press.

Harada, T., Yoshimoto, F., \& Moriyama, M. (1999). An aesthetic curve in the field of industrial design. Proc. IEEE Symposium on Visual Language, Tokyo (pp. 38-47).

Kanaya, I., Nakano, Y., \& Sato, K. (2003). Simulated designer's eyes: Classification of aesthetic surfaces. Proc. VSMM 2003, Montreal (pp. 289-29).

Keller, H. B. (1968). Numerical methods for two-point boundary-value problems. Blaisdell, Waltham, Massachusetts.

Miura, K. T., \& Gobithaasan, R. U. (2014). Aesthetic curves and surfaces in Computer Aided Geometric Design. Int. Journal of Automation Technology, in press.

Miura, K. T., Shibuya, D., Gobithaasan, R. U., \& Usuki, S. (2013). Designing Log-aesthetic Splines with G2 Continuity. Computer-Aided Design and Applications, 10(6), 1021-1032. http://dx.doi.org/10.3722/cadaps.2013.1021-1032

Miura, K. T. (2005). Derivation of general formula of aesthetic curves. Proc. of 8th Int. Conf. on Humans \& Computers, Tokyo (pp. 166-171).

Miura, K. T., Shirahata, R., Agari, S., Usuki, S., \& Gobithaasan, R. U. (2012). Variational formulation of the $\log$-aesthetic surface and development of discrete surface filters. Computer-Aided Design and Applications, 9(6), 901-914. http://dx.doi.org/10.3722/cadaps.2012.901-914

Pugh, S. (1991). Total Design. Great Britain: Addison-Wesley Publishing Company.

Yoshida, N., \& Saito, T. (2006). Interactive aesthetic curve segments. Visual Computers, 22, 896-905.

\section{Copyrights}

Copyright for this article is retained by the author(s), with first publication rights granted to the journal.

This is an open-access article distributed under the terms and conditions of the Creative Commons Attribution license (http://creativecommons.org/licenses/by/3.0/). 